Washington University School of Medicine Digital Commons@Becker

Open Access Publications

2017

\title{
Independent test of a model to predict severe acute esophagitis
}

\author{
Ellen X. Huang \\ Johns Hopkins University \\ Clifford G. Robinson \\ Washington University School of Medicine in St. Louis \\ Alerson Molotievschi \\ Washington University School of Medicine in St. Louis \\ Jeffrey D. Bradley \\ Washington University School of Medicine in St. Louis \\ Joseph O. Deasy \\ Memorial Sloan Kettering Cancer Center
}

See next page for additional authors

Follow this and additional works at: https://digitalcommons.wustl.edu/open_access_pubs

Please let us know how this document benefits you.

\section{Recommended Citation}

Huang, Ellen X.; Robinson, Clifford G.; Molotievschi, Alerson; Bradley, Jeffrey D.; Deasy, Joseph O.; and Oh, Jung Hun, "Independent test of a model to predict severe acute esophagitis." Advances in radiation oncology. 2, 1. 37-43. (2017).

https://digitalcommons.wustl.edu/open_access_pubs/5674

This Open Access Publication is brought to you for free and open access by Digital Commons@Becker. It has been accepted for inclusion in Open Access Publications by an authorized administrator of Digital Commons@Becker. For more information, please contact vanam@wustl.edu. 


\section{Authors}

Ellen X. Huang, Clifford G. Robinson, Alerson Molotievschi, Jeffrey D. Bradley, Joseph O. Deasy, and Jung Hun Oh 


\title{
Scientific Article
}

\section{Independent test of a model to predict severe acute esophagitis}

\section{Ellen X. Huang PhD ${ }^{a}$, Clifford G. Robinson MD ${ }^{b}$, Alerson Molotievschi MD ${ }^{b}$, Jeffrey D. Bradley MD ${ }^{b}$,

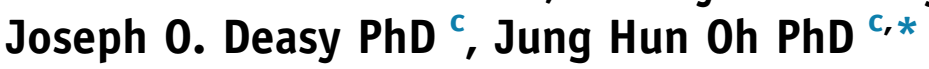

\author{
${ }^{a}$ Department of Radiation Oncology and Molecular Sciences, Johns Hopkins University School of \\ Medicine, Baltimore, Maryland \\ ${ }^{\mathrm{b}}$ Department of Radiation Oncology, Washington University School of Medicine, Saint Louis, \\ Missouri \\ ${ }^{\mathrm{c}}$ Department of Medical Physics, Memorial Sloan Kettering Cancer Center, New York, New York
}

Received 15 August 2016; received in revised form 28 October 2016; accepted 9 November 2016

\begin{abstract}
Purpose: Treatment planning factors are known to affect the risk of severe acute esophagitis during thoracic radiation therapy. We tested a previously published model to predict the risk of severe acute esophagitis on an independent data set.

Methods and materials: The data set consists of data from patients who had recoverable treatment plans and received definitive radiation therapy for non-small cell carcinoma of the lung at a single institution between November 2004 and January 2010. Complete esophagus dose-volume and available clinical information was extracted using our in-house software. The previously published model was a logistic function with a combination of mean esophageal dose and use of concurrent chemotherapy. In addition to testing the previous model, we used a novel, machine learning-based method to build a maximally predictive model.

Results: Ninety-four patients $(81.7 \%)$ developed Common Terminology Criteria for Adverse Events, Version 4, Grade 2 or more severe esophagitis (Grade 2: $\mathrm{n}=79$ and Grade 3: $\mathrm{n}=15$ ). Univariate analysis revealed that the most statistically significant dose-volume parameters included percentage of esophagus volume receiving $\geq 40$ to $60 \mathrm{~Gy}$, minimum dose to the highest $20 \%$ of esophagus volume (D20) to D35, and mean dose. Other significant predictors included concurrent chemotherapy and patient age. The previously published model predicted risk effectively with a Spearman's rank correlation coefficient $\left(\mathrm{r}_{\mathrm{s}}\right)$ of $0.43(P<.001)$ with good calibration (HosmerLemeshow goodness of fit: $P=.537)$. A new model that was built from the current data set found the same variables, yielding an $\mathrm{r}_{\mathrm{s}}$ of $0.43(P<.001)$ with a logistic function of $0.0853 \times$ mean esophageal dose $[\mathrm{Gy}]+1.49 \times$ concurrent chemotherapy [1/0] -1.75 and Hosmer-
\end{abstract}

Sources of support: This research was supported by the National Institutes of Health (Grant number R01 CA85181) and in part through the National Institutes of Health/National Cancer Institute Cancer Center Support Grant (Grant number P30 CA008748).

Conflicts of interest: None.

Supplementary material related to this article can be found at http://dx.doi.org/10.1016/j.adro.2016.11.003.

* Corresponding author. Department of Medical Physics, Memorial Sloan Kettering Cancer Center, 1275 York Ave., New York, NY 10065

E-mail address: ohj@mskcc.org (J.H. Oh) 
Lemeshow $P=.659$. A novel preconditioned least absolute shrinkage and selection operator method yielded an average $r_{s}$ of 0.38 on 100 bootstrapped data sets.

Conclusions: The previously published model was validated on an independent data set and determined to be nearly as predictive as the best possible two-parameter logistic model even though it overpredicted risk systematically. A novel, machine learning-based model using a bootstrapping approach showed reasonable predictive power.

(C) 2016 the Authors. Published by Elsevier Inc. on behalf of the American Society for Radiation Oncology. This is an open access article under the CC BY-NC-ND license (http:// creativecommons.org/licenses/by-nc-nd/4.0/).

\section{Introduction}

Severe acute esophagitis (AE) is a common, doselimiting side effect of radiation therapy for patients with non-small cell lung cancer. When it occurs, AE often peaks in the first few weeks of a course of radiation therapy. ${ }^{1-3}$ Patient-related, tumor-related, and treatment-related risk factors ${ }^{3-8}$ that have been reported to be statistically associated with the incidence or severity of AE include age, tumor nodal stage, concurrent chemotherapy, and body mass index. Two recent reviews ${ }^{9,10}$ summarized the dosimetric predictors. Rose et $\mathrm{al}^{9}$ systematically reviewed 18 published studies of patients with non-small cell lung cancer who had radiation-induced esophagitis. ${ }^{3-7,11-23}$ Eleven studies specifically assessed AE, and the other studies assessed acute and chronic radiation-induced esophagitis together. Five dosimetric parameters were identified as predictive of AE with or without chemotherapy: mean esophageal dose (MED), maximal esophageal dose, percentage of esophagus volume receiving $\geq 20$ Gy (V20), V35, and V60. In a QUANTEC review article, Werner-Wasik et al ${ }^{10}$ noted disparities in the dosimetric parameters that were identified as most predictive of AE.

Our recently published analysis ${ }^{24}$ of a large cohort of single institution data suggested that a two-variable logistic model based on MED and use of concurrent chemotherapy robustly predicts risk of $\mathrm{AE}$ in a combined data set that includes data from patients at our institution between 1991 and 2000 and from the Radiation Therapy Oncology Group (RTOG) 93-11 trial. The main purpose of this study is to test the published two-variable model on a new, independent data set; update the model for clinical use; and propose a novel machine learning-based predictive model.

\section{Methods and materials}

\section{Patient cohort}

This study received approval from an institutional review board, and all patients provided informed consent. All patients who were treated at Washington University School of Medicine in St. Louis between November 2004 and January 2010, who received a minimal target dose of 46.2 Gy, and whose dose distributions were computed with modern dosimetry (ie, collapsed cone algorithm used in the Pinnacle treatment planning system as opposed to water-based methods) were included in this study. General patient characteristics are provided in Table 1.

\section{Treatment characteristics}

Patients were treated either with two-course radiation therapy $(73.9 \%)$ or a full course of image guided radiation therapy $(26.1 \%)$. Two-course radiation therapy consisted of parallel opposed (anterior-posterior and posterioranterior) beams for a few weeks, followed by off-cord oblique beams to spare part of the esophagus. Nine patients $(7.8 \%)$ received neoadjuvant chemotherapy, 85 patients $(73.9 \%)$ received concurrent chemotherapy, and 54 patients $(47.0 \%)$ received adjuvant chemotherapy. Five patients $(4.4 \%)$ received both neoadjuvant and concurrent chemotherapy, but $41(35.7 \%)$ received both concurrent and adjuvant chemotherapy. One patient received neoadjuvant, concurrent, and adjuvant chemotherapy.

\section{Acute esophagitis events}

Complications were defined as Common Terminology Criteria for Adverse Events, Version 4 (CTCAE V4.0), Grade $\geq 2$ events (ie, events that required medical attention or care). Ninety-four patients $(81.7 \%)$ developed severe esophagitis, 79 of whom $(68.7 \%)$ had Grade 2 complications and 15 of whom (13\%) developed Grade 3 complications. No Grade 4 or 5 events were reported.

\section{Dose-volume parameters}

A wide range of esophagus dose-volume parameters was extracted for modeling. The entire length of the esophagus was contoured. $\mathrm{D}_{\mathrm{x}}$ is defined as the minimum dose to the $\mathrm{x} \%$ volume of the esophagus receiving the highest doses. $\mathrm{V}_{\mathrm{x}}$ is the percentage volume of the esophagus receiving at least $\mathrm{x} \mathrm{Gy} . \mathrm{MOH}_{\mathrm{x}}$ is defined as the mean dose of the $\mathrm{x} \%$ volume of the esophagus receiving 
Table 1 Patient characteristics

\begin{tabular}{|c|c|c|c|c|}
\hline Parameter & All patients $(\mathrm{n}=115)$ & Grade $<2(\mathrm{n}=21)$ & Grade $\geq 2(n=94)$ & $P$ value \\
\hline Age (years), mean (range) & $64.2(37-86)$ & 69.9 & 62.9 & .005 \\
\hline Gender, n (\%) & & & & .950 \\
\hline Male & $50 / 115(43.5 \%)$ & 9 & 41 & \\
\hline Female & $65 / 115(56.5 \%)$ & 12 & 53 & \\
\hline Race, n (\%) & & & & .771 \\
\hline White & $76 / 115(66.1 \%)$ & 14 & 62 & \\
\hline Black & $36 / 115(31.3 \%)$ & 6 & 30 & \\
\hline Other & $3 / 115(2.6 \%)$ & 1 & 2 & \\
\hline $\begin{array}{l}\text { Performance status } \\
\text { (ECOG/ZUBROD SCORE), n (\%) }\end{array}$ & & & & .969 \\
\hline 0 & $48 / 115(41.7 \%)$ & 8 & 40 & \\
\hline 1 & $50 / 115(43.5 \%)$ & 9 & 41 & \\
\hline 2 & $13 / 115(11.3 \%)$ & 2 & 11 & \\
\hline Unknown & $4 / 115(3.5 \%)$ & 2 & 2 & \\
\hline Weight loss, n (\%) & & & & .235 \\
\hline$<10 \%$ & $87 / 115(75.6 \%)$ & 18 & 69 & \\
\hline$>10 \%$ & $28 / 115(24.4 \%)$ & 3 & 25 & \\
\hline Tumor stage, n (\%) & & & & .696 \\
\hline$\geq 2$ & $86 / 115(74.8 \%)$ & 15 & 71 & \\
\hline$<2$ & $29 / 115(25.2 \%)$ & 6 & 23 & \\
\hline Primary histology, n (\%) & & & & .272 \\
\hline Squamous cell & $34 / 115(29.6 \%)$ & 7 & 27 & \\
\hline Adenocarcinoma & $73 / 115(63.4 \%)$ & 11 & 62 & \\
\hline Non-small cell & $8 / 115(7.0 \%)$ & 3 & 5 & \\
\hline Treatment time (days), mean (range) & $48.6(31-68)$ & 47.9 & 48.7 & .836 \\
\hline Fraction size (Gy), median (range) & $2.0(1.8-2.5)$ & 2 & 2 & .296 \\
\hline Chemotherapy, n (\%) & & 23 & 125 & .062 \\
\hline Pre-radiation therapy & $9 / 115(7.8 \%)$ & 0 & 9 & \\
\hline Concurrent & $85 / 115(73.9 \%)$ & 10 & 75 & \\
\hline Post-radiation therapy & $54 / 115(47.0 \%)$ & 13 & 41 & \\
\hline Smoker, n (\%) & & & & .488 \\
\hline Current & $42 / 115(36.5 \%)$ & 7 & 35 & \\
\hline Former & $68 / 115(59.1 \%)$ & 14 & 54 & \\
\hline Never & $5 / 115(4.4 \%)$ & 0 & 5 & \\
\hline \multicolumn{5}{|l|}{ Mode of radiation therapy, $\mathrm{n}(\%)$} \\
\hline IMRT & $30 / 115(26.1 \%)$ & 5 & 25 & .793 \\
\hline 3DCRT & $85 / 115(73.9 \%)$ & 16 & 69 & \\
\hline
\end{tabular}

The $\chi^{2}$ test was used for categorical variables, and Wilcoxon rank-sum test was used for continuous variables.

the highest doses, and $\mathrm{MOC}_{\mathrm{x}}$ is similarly defined as the mean dose of the coldest $\mathrm{x} \%$ volume of the organ. $\mathrm{MOH}_{\mathrm{x}}$ and $\mathrm{MOC}_{\mathrm{x}}$ were included in the model because they could potentially be more robust predictors of dosimetric effect than $\mathrm{D}_{\mathrm{x}}$ and $\mathrm{V}_{\mathrm{x}}$ due to their averaging characteristic compared with focusing on a single dose-volume histogram point. Thus, the dosimetric parameters of esophagus that were analyzed included D5 to D100, V5 to V100, MOH5 to MOH100, and MOC5 to MOC100 (in increments of 5), mean dose, International Commission on Radiation Units and Measurements maximum dose (ie, highest mean dose to any cuboid volume at least $1.5 \mathrm{~cm}$ on a side), and International Commission on Radiation Units and Measurements minimum dose (ie, lowest mean dose to any cuboid volume at least $1.5 \mathrm{~cm}$ on a side). Clinical variables included age, sex, race, Karnofsky performance status, weight loss, chemotherapy (neoadjuvant or concurrent), histology, and clinical stage.

\section{Statistical analysis and modeling}

Our first step was to test the previously published AE model $^{24}$ on the current cohort. To find a new model on the current cohort, univariate logistic analysis was performed for each available variable and $r_{s}$ was computed to assess univariate correlation with risk of AE. Finally, multivariate logistic regression modeling was conducted as designed by Huang et al. ${ }^{24}$ In this approach, during leaveone-out cross validation (LOOCV), the most frequently generated model was chosen as a final predictive model, and all samples were input into the model to calculate its predictive power. 


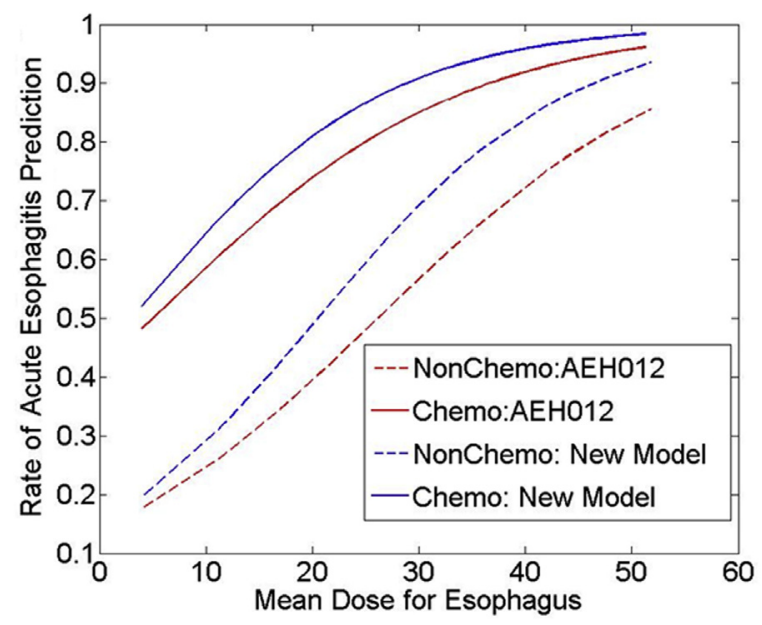

Fig. 1 Estimated dose response curves according to the AEH012 model (red lines) versus the new model (blue lines) for Grade 2 or greater acute esophagitis with or without concurrent chemotherapy.

In addition, as described in the supplementary material, we designed a novel two-step process to produce predictive models on the basis of machine learning methods. In the first step, we filtered irrelevant variables (ie, those with low univariate correlations) and reduced competing, redundant variables, which left 12 variables (D10, D25, D40, V20, V50, V65, MOH15, MOH45, age, grade, concurrent chemo, and mean esophagus dose) for input into the next step. A preconditioning step that was inspired by a study by Debashis et $\mathrm{al}^{25}$ was applied next. The goal of preconditioning is to introduce a more useful response variable of outcomes than the observed binary outcomes. This is useful if the model to generate preconditioning has validity and is complementary to the next modeling stage. Finally, filtered variables with preconditioned outcomes were fed into a least absolute shrinkage and selection operator (LASSO) modeling process. We generated 100 bootstrapped data sets with replacement. At each iteration, a logistic regression model with forward variable selection was generated and its outputs (normal tissue complication probability [NTCP]) were used as a response variable in the LASSO regression modeling with 12 variables as independent variables. Predictive LASSO outputs were used to assess correlation with risk of AE. After completion of the entire process, the predictive performance was averaged.

\section{Results}

\section{Test of the previously published AE model}

The previously published two-variable model to predict risk of $\mathrm{AE}^{24}$ that is shown below uses the variables of

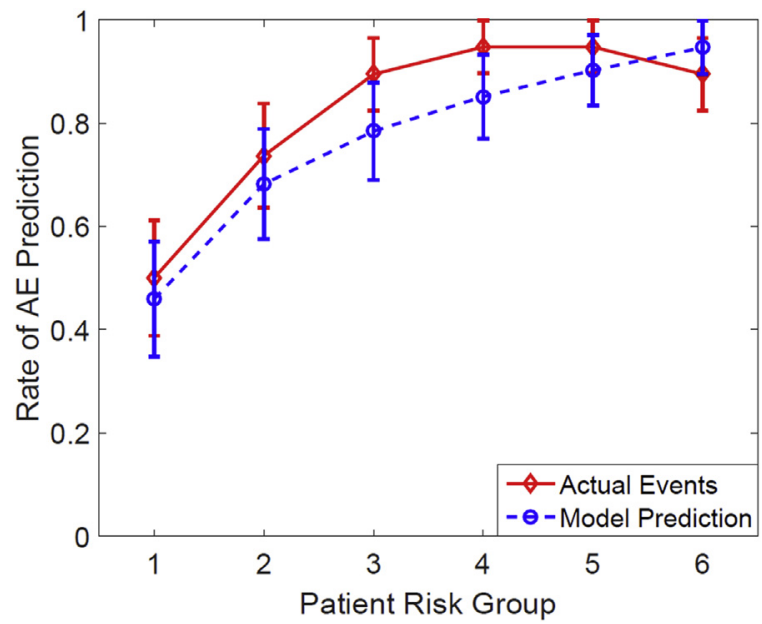

Fig. 2 Mean predicted rates of acute esophagitis versus observed rates for patients. The patients were binned in accordance with the predicted risk of acute esophagitis by the AEH012 two-variable model. The mean predicted and observed event rates in each bin are (predicted risk; events/pts) $(0.4590$, 10/20), (0.6820, 14/19), (0.7843, 17/19), (0.8515, 18/19), (0.9023, 18/19), (0.9463, 17/19).

mean esophagus dose and concurrent chemotherapy (ConChemo $=1$ if given, and $=0$ if not given):

$\mathrm{NTCP}_{\mathrm{AEH} 012}=\frac{1}{1+\exp \left(-\mathrm{x}_{\mathrm{AEH} 012}\right)}$,

where

$\mathrm{x}_{\mathrm{AEH} 012}=0.0688 \times$ MED $+1.5 \times$ ConChemo -3.13 .

The model performed well on the current cohort with an $\mathrm{r}_{\mathrm{s}}$ of $0.43(P<.001)$ and good calibration (HosmerLemeshow goodness of fit: $P=.537$, degrees of freedom $=6$ ). Figure 1 shows the estimated logistic regression curves as a function of mean esophageal dose with and without concurrent chemotherapy for the AEH012 model. The model-predicted incidence of $\mathrm{AE}$ versus observed incidence is displayed in Figure 2, with patients divided into 6 equal bins and grouped in accordance with the model-predicted risk.

\section{Univariate logistic regression}

Mean, median, and range values for representative $D_{x}$, $\mathrm{V}_{\mathrm{x}}$, and $\mathrm{MOH}_{\mathrm{x}}$ variables for the esophagus are listed in Table 2. Eighteen variables with $\mathrm{r}_{\mathrm{s}}>0.3$ and significance of $P<.0005$ are listed in Supplemental Table 1 .

\section{Multivariate risk modeling}

A new two-variable model on the new cohort was suggested with use of the model building process that was employed by Huang et $\mathrm{al}^{24}$ on the basis of LOOCV. Interestingly, the most frequently generated model during LOOCV consisted of the same combination of MED and 
Table 2 Summary of dosimetric statistics for the esophagus, mean (SD)

\begin{tabular}{|c|c|c|c|c|}
\hline & Variable & Grade $<2$ & Grade $\geq 2$ & $P$-value \\
\hline \multirow[t]{8}{*}{$\mathrm{D}_{\mathrm{x}}(\mathrm{Gy})$} & D10 & 49.1 (20.9) & $64.4(9.9)$ & .0004 \\
\hline & D20 & $41.1(22.2)$ & $59.3(13.3)$ & .0003 \\
\hline & D30 & $33.8(22.6)$ & $52.6(17.6)$ & .0005 \\
\hline & D40 & $25.2(18.9)$ & $41.5(22.2)$ & .0023 \\
\hline & D50 & $16.3(15.7)$ & $29.2(22.7)$ & .0291 \\
\hline & D60 & $11.0(13.3)$ & 17.9 (19.6) & .1840 \\
\hline & D70 & $7.3(9.7)$ & $9.1(12.8)$ & .7860 \\
\hline & D80 & $2.9(3.3)$ & $4.0(7.4)$ & .9049 \\
\hline \multirow[t]{7}{*}{$\mathrm{V}_{\mathrm{x}}(\%)$} & V10 & $48.3(24.4)$ & $57.6(16.1)$ & .1205 \\
\hline & V20 & $40.1(22.4)$ & $52.6(17.0)$ & .0293 \\
\hline & V30 & $30.6(20.4)$ & $48.3(17.2)$ & .0004 \\
\hline & V40 & $24.8(19.6)$ & 43.7 (17.6) & .0001 \\
\hline & V50 & $18.7(18.5)$ & $37.7(18.0)$ & .0001 \\
\hline & V60 & $12.3(15.0)$ & $28.5(16.9)$ & .0001 \\
\hline & V70 & $2.8(7.1)$ & $8.0(12.6)$ & .0871 \\
\hline \multirow{8}{*}{$\mathrm{MOH}_{\mathrm{x}}(\mathrm{Gy})$} & MOH10 & $54.3(19.0)$ & $66.6(8.6)$ & .0018 \\
\hline & MOH20 & 49.7 (19.8) & $64.3(9.5)$ & .0004 \\
\hline & МOH30 & $45.6(20.3)$ & $61.6(10.9)$ & .0003 \\
\hline & MOH40 & $41.5(20.1)$ & $58.0(12.5)$ & .0003 \\
\hline & MOH50 & 37.2 (18.9) & $53.5(13.8)$ & .0003 \\
\hline & MOH60 & $33.2(17.3)$ & $48.4(14.2)$ & .0005 \\
\hline & MOH70 & $29.8(16.0)$ & $43.2(13.8)$ & .0008 \\
\hline & MOH80 & $26.5(14.5)$ & $38.3(12.9)$ & .0013 \\
\hline \multirow[t]{3}{*}{ Other (Gy) } & $\begin{array}{l}\text { Mean } \\
\text { Dose }\end{array}$ & 21.8 (11.6) & $31.6(10.4)$ & .0011 \\
\hline & $\begin{array}{l}\text { Minimum } \\
\text { Dose }\end{array}$ & 0.9 (1.6) & $0.8(1.4)$ & .6263 \\
\hline & $\begin{array}{l}\text { Maximum } \\
\text { Dose }\end{array}$ & $62.9(15.3)$ & $69.7(8.0)$ & .0767 \\
\hline
\end{tabular}

$\mathrm{D}_{\mathrm{x}}$, minimum dose to the $\mathrm{x} \%$ volume of the esophagus receiving the highest doses; $\mathrm{MOH}_{\mathrm{x}}$, mean dose of the $\mathrm{x} \%$ volume of the esophagus receiving the highest doses; $\mathrm{V}_{\mathrm{x}}$, percentage volume of the esophagus receiving at least $\mathrm{x}$ Gy.

ConChemo as the previous model. The resulting fitted risk of $\mathrm{AE}$ as a logistic function is given by:

$\mathrm{NTCP}=\frac{1}{1+\exp (-\mathrm{x})}$,

where $\mathrm{x}=0.0853 \times$ MED $+1.49 \times$ ConChemo -1.75 .

The $\mathrm{r}_{\mathrm{s}}$ of this risk model on the current cohort was 0.43 $(P<.001$; note that it was not cross-validated). The Hosmer-Lemeshow test showed a good fit in this model $(P=.659$, degrees of freedom $=6)$. Figure 1 shows the estimated logistic regression curves as a function of MED with and without chemotherapy. Figure 3 compares the model-predicted incidence of $\mathrm{AE}$ and the observed incidence with patients divided into 6 equal bins and grouped in accordance with the model-predicted risk. For ConChemo and MED, the odds ratio of the observed AE rate between the one-third of patients at high risk and the onethird of patients at low risk was $3.59(P=.037)$ and 1.08 $(P=.003)$, respectively, which is another indicator that

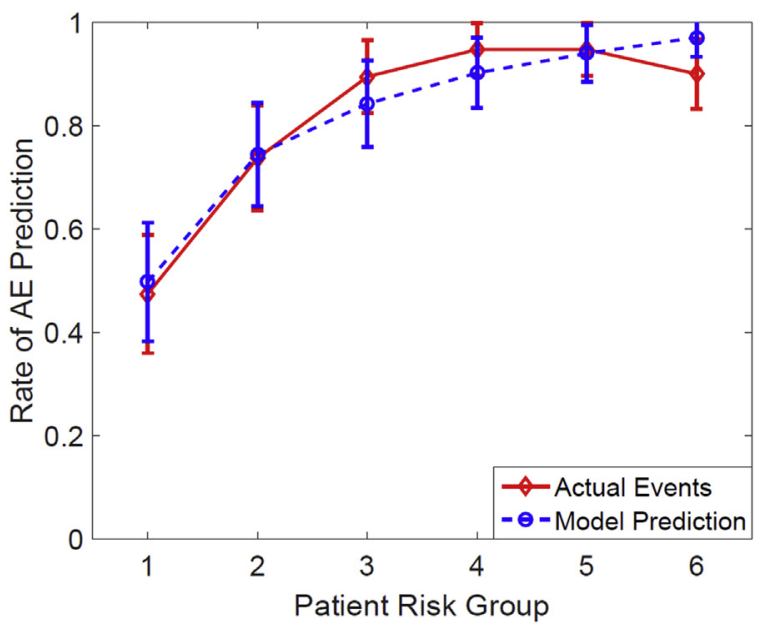

Fig. 3 Mean predicted rates of acute esophagitis versus observed rates for patients. The patients were binned in accordance with the predicted risk of acute esophagitis by the best fit two-variable model. The mean predicted and observed event rates in each bin are (predicted risk; events/pts): $(0.5059,10 / 20)$, (0.7504, 14/19), (0.8465, 17/19), (0.9034, 18/19), (0.9419, 18/ 19), $(0.9715,17 / 19)$.

the model usefully distinguishes high-risk patients from low-risk patients.

\section{Model-based preconditioning LASSO regression model}

After filtering out irrelevant and redundant variables, the resulting 12 variables were entered in a logistic regression model with forward variable selection on a bootstrapped data set with replacement. Its output (ie, preconditioned outcomes) was entered in a LASSO regression model. After selecting a tuning parameter in LASSO using 10-fold cross validation, the final model was tested with samples that were not included in the bootstrapped data set. We iterated this process 100 times. An example of the LASSO model fit is shown in Supplemental Figure 1. Our approach, coupled with logistic regression and LASSO, performed better than a logistic regression model with forward variable selection alone, which was used to find the best model as shown in the above section with an average $r_{s}$ of 0.38 and 0.34 , respectively.

\section{Discussion}

\section{Similarity of current patient cohort to previous cohort}

When comparing the current cohort with the previous AE study cohort, there was no difference in terms of age (mean of 65.3 years in the current cohort vs 64.2 years in the previous cohort) and fraction size (1.8-2.5 Gy). In terms of sex, the current cohort included more female 
Table 3 Performance comparison of the model AEH012 with the new model for acute esophagitis prediction, both on the new data set (note that both models were not crossvalidated)

\begin{tabular}{lll}
\hline Model & Spearman Correlation & AUC \\
\hline New model & $0.43(p<0.001)$ & 0.78 \\
AEH012 & $0.43(p<0.001)$ & 0.78 \\
\hline
\end{tabular}

AUC, area under the receiver operating characteristic curve.

(56.5\%) than male patients $(43.5 \%)$, but the previous cohort included an almost even number of male and female patients (51.9\% vs $48.1 \%$, respectively). With regard to race, the current cohort was categorized as white $66.1 \%$, black $31.3 \%$, and other $2.6 \%$ compared with the previous cohort, which had white $74.7 \%$, black $24 \%$, and other $1.3 \%$. As for chemotherapy, $73.9 \%$ of patients in the current cohort received concurrent chemotherapy, but only $36.7 \%$ of the previous cohort had concurrent chemotherapy. The increase in use of concurrent chemotherapy is the largest differentiator between the cohorts. Despite this, the models explicitly account for concurrent chemotherapy on a patient-by-patient basis; therefore, the difference does not invalidate the test.

\section{Acute esophagitis events}

A difference between this study and the previous study is that in the new cohort, $\mathrm{AE}$ events were scored on the basis of CTCAE V4.0 whereas the previously studied cohort was scored using RTOG AE criteria. Despite this difference, RTOG Grades 1 and 2 combined (ie, complications that do not require medical intervention) were judged as comparable with CTCAE V4.0 Grade 2. This correspondence has been used here.

\section{Treatment characteristics}

Patients in the previously studied cohort were treated completely with two-course radiation therapy, which included parallel opposed, anterior-posterior/posterioranterior beams, for the first few weeks, followed by offcord oblique beams to spare part of the esophagus and spinal cord. In the previous cohort, 54\% (128 of 237 patients) received sequential or concurrent chemotherapy. Patients in the current cohort were treated with 3-dimensional conformal radiation therapy or image guided radiation therapy techniques, and all patients received chemotherapy before, during, or after radiation therapy.

\section{Statistical analysis}

In terms of univariate analysis, the percentage volume of the esophagus receiving a range of doses including 60 ,
50, 40, and 30 Gy; mean dose; and concurrent chemotherapy was highly correlated with $\mathrm{AE}$ events for both cohorts. The best two-variable model included mean dose of the esophagus and concurrent chemotherapy.

\section{Model comparison}

To compare the performance of the best model derived from the previously studied cohort and the best model derived from the current cohort, each model was applied to the current cohort. The predictive power for actual AE events is shown in Table 3. The ability to predict $\mathrm{AE}$ events with both models was almost identical in terms of $r_{s}$ and the area under the receiver operating characteristic curve. In addition, we designed a novel preconditioning LASSO regression model to predict the risk of developing AE. For an unbiased assessment of the model, a bootstrapping approach was used, which resulted in a good level of predictive power.

\section{Conclusions}

The previously published model to determine the risk probability of severe AE (as indicated by medical management) was validated on a new, independent data set and showed to be nearly as predictive as the best possible two-variable logistic model derived from the new data set. Although we judged the model to be validated and statistically significant, it is important to further test the model with other institutions' data.

\section{References}

1. Bradley J, Movsas B. Radiation esophagitis: Predictive factors and preventive strategies. Semin Radiat Oncol. 2004;14:280-286.

2. Bradley J, Movsas B. Radiation pneumonitis and esophagitis in thoracic irradiation. Cancer Treat Res. 2006;128:43-64.

3. Bradley J, Deasy JO, Bentzen S, El-Naqa I. Dosimetric correlates for acute esophagitis in patients treated with radiotherapy for lung carcinoma. Int J Radiat Oncol Biol Phys. 2004;58:1106-1113.

4. Ahn SJ, Kahn D, Zhou S, et al. Dosimetric and clinical predictors for radiation-induced esophageal injury. Int $J$ Radiat Oncol Biol Phys. 2005;61:335-347.

5. Belderbos J, Heemsbergen W, Hoogeman M, Pengel K, Rossi M, Lebesque J. Acute esophageal toxicity in non-small cell lung cancer patients after high dose conformal radiotherapy. Radiother Oncol. 2005;75:157-164.

6. Chapet O, Kong FM, Lee JS, Hayman JA, Ten Haken RK. Normal tissue complication probability modeling for acute esophagitis in patients treated with conformal radiation therapy for non-small cell lung cancer. Radiother Oncol. 2005;77:176-181.

7. Patel AB, Edelman MJ, Kwok Y, Krasna MJ, Suntharalingam M. Predictors of acute esophagitis in patients with non-small-cell lung carcinoma treated with concurrent chemotherapy and hyperfractionated radiotherapy followed by surgery. Int J Radiat Oncol Biol Phys. 2004;60:1106-1112.

8. Werner-Wasik M. Treatment-related esophagitis. Semin Oncol. 2005;32:S60-S66. 
9. Rose J, Rodrigues G, Yaremko B, Lock M, D’Souza D. Systematic review of dose-volume parameters in the prediction of esophagitis in thoracic radiotherapy. Radiother Oncol. 2009;91: 282-287.

10. Werner-Wasik M, Yorke E, Deasy J, Nam J, Marks LB. Radiation dose-volume effects in the esophagus. Int J Radiat Oncol Biol Phys. 2010;76:S86-S93.

11. Kahn D, Zhou S, Ahn SJ, et al. "Anatomically-correct" dosimetric parameters may be better predictors for esophageal toxicity than are traditional CT-based metrics. Int J Radiat Oncol Biol Phys. 2005;62: 645-651.

12. Wei X, Liu HH, Tucker SL, et al. Risk factors for acute esophagitis in non-small-cell lung cancer patients treated with concurrent chemotherapy and three-dimensional conformal radiotherapy. Int $J$ Radiat Oncol Biol Phys. 2006;66:100-107.

13. Qiao WB, Zhao YH, Zhao YB, Wang RZ. Clinical and dosimetric factors of radiation-induced esophageal injury: radiation-induced esophageal toxicity. World J Gastroenterol. 2005;11:2626-2629.

14. Singh AK, Lockett MA, Bradley JD. Predictors of radiation-induced esophageal toxicity in patients with non-small-cell lung cancer treated with three-dimensional conformal radiotherapy. Int J Radiat Oncol Biol Phys. 2003;55:337-341.

15. Kim TH, Cho KH, Pyo HR, et al. Dose-volumetric parameters of acute esophageal toxicity in patients with lung cancer treated with three-dimensional conformal radiotherapy. Int J Radiat Oncol Biol Phys. 2005;62:995-1002.

16. Choy H, LaPorte K, Knill-Selby E, Mohr P, Shyr Y. Esophagitis in combined modality therapy for locally advanced non-small cell lung cancer. Semin Radiat Oncol. 1999;9:90-96.

17. Werner-Wasik M, Langer C, Movsas B. Amifostine in chemoradiation therapy for non-small cell lung cancer: review of experience and design of a phase II trial assessing subcutaneous and intravenous bolus administration. Semin Oncol. 2005;32: S105-S108.

18. Maguire PD, Sibley GS, Zhou SM, et al. Clinical and dosimetric predictors of radiation-induced esophageal toxicity. Int J Radiat Oncol Biol Phys. 1999;45:97-103.

19. Rosenman JG, Halle JS, Socinski MA, et al. High-dose conformal radiotherapy for treatment of stage IIIA/IIIB non-small-cell lung cancer: technical issues and results of a phase I/II trial. Int J Radiat Oncol Biol Phys. 2002;54:348-356.

20. Takeda K, Nemoto K, Saito H, Ogawa Y, Takai Y, Yamada S. Dosimetric correlations of acute esophagitis in lung cancer patients treated with radiotherapy. Int J Radiat Oncol Biol Phys. 2005;62: 626-629.

21. Takeda K, Nemoto K, Saito H, Ogawa Y, Takai Y, Yamada S. Predictive factors for acute esophageal toxicity in thoracic radiotherapy. Tohoku J Exp Med. 2006;208:299-306.

22. Hirota S, Tsujino K, Endo M, et al. Dosimetric predictors of radiation esophagitis in patients treated for non-small-cell lung cancer with carboplatin/paclitaxel/radiotherapy. Int J Radiat Oncol Biol Phys. 2001;51:291-295.

23. Langer CJ, Movsas B, Hudes R, et al. Induction paclitaxel and carboplatin followed by concurrent chemoradiotherapy in patients with unresectable, locally advanced non-small cell lung carcinoma: report of Fox Chase Cancer Center study 94-001. Semin Oncol. 1997;24. S12-89-S12-95.

24. Huang EX, Bradley JD, El Naqa I, et al. Modeling the risk of radiation-induced acute esophagitis for combined Washington University and RTOG trial 93-11 lung cancer patients. Int J Radiat Oncol Biol Phys. 2012;82:1674-1679.

25. Paul D, Bair E, Hastie T, et al. "Pre-conditioning" for feature selection and regression in high-dimensional problems. Annals of Statistics. 2008;36:1595-1618. 